\title{
Síndrome hipotónico del recién nacido
}

\author{
ALICIA NÚÑEZ F. ${ }^{1}$, JUAN ARÁNGUIZ R. ${ }^{1}$, JAVIER KATTAN S. ${ }^{2}$, RAÚL ESCOBAR H. ${ }^{3}$
}

1. Residente Neurología Pediátrica, Departamento de Pediatría. Facultad de Medicina. Pontificia Universidad Católica de Chile. Santiago, Chile.

2. Neonatólogo, Departamento de Pediatría. Facultad de Medicina. Pontificia Universidad Católica de Chile. Santiago, Chile.

3. Neurólogo Pediatra, Departamento de Pediatría. Facultad de Medicina. Pontificia Universidad Católica de Chile. Santiago, Chile.

\begin{abstract}
\section{Hypotonic syndrome in the newborn}

Background: The Newborn Hypotonic Syndrome (NHS) is a clinical entity that presents up to 28 days after birth. The main symptom is a significant decrease in muscular tone, but its severity is determined by the lack of muscular strength. NHS is a relatively frequent entity, so it becomes an important diagnosis problem. There is few information in literature regarding its incidence. Method: Retrospective study performed between May 2000 - April 2006, including patients with diagnosis of NHS in a Neonatal Intesive Care Unit. Results: 2158 newborns, of which 113 (5.2\%) had NHS. 83\% of cases were attributed to central causes, such as hipoxic-isquemic encephalopathy (49\%) and genetic disorders (15\%). $17 \%$ of cases corresponded to peripheral causes, including hypermagnesemia (68\%) and myopathic diseases (21\%). Conclusions: 1) NHS is a relatively frequent clinical entity; 2) Central causes are the most prevalent; 3) It is possible to study the etiology of NHS with a systematic approach.
\end{abstract}

(Key words: neonatal hypotonia, floppy newborn, neonate).

Rev Chil Pediatr 2008; 79 (2): 146-151

\section{RESUMEN}

Introducción: El síndrome hipotónico del recién nacido (SHRN) es un cuadro clínico que se presenta hasta los 28 días de vida extrauterina. Su síntoma definitorio es la disminución significativa en tono muscular, pero su gravedad se relaciona a asociación a falta de fuerzas. Es un cuadro aparentemente poco frecuente en este grupo etáreo, pero que plantea un problema diagnostico importante. Existe escasa información en la literatura respecto a su frecuencia real. Objetivo: Evaluar la incidencia de SHRN, su etiología y aprobación diagnóstica. Método: Estudio retrospectivo con revisión de fichas clínicas, desde mayo 2000 a abril 2006, incluyendo pacientes ingresados con diagnostico de SHRN a unidad de intermedio-intensivo de servicio de recién-nacidos de un hospital universitario (SRNU). Resultados: El número total de RN ingresados a SRNU fue 2158, en 5,2\% (113) el motivo de ingreso, principal o secundario, correspondió a

Trabajo recibido el 25 de julio de 2007, devuelto para corregir el 21 de noviembre de 2007, segunda versión el 02 de enero de 2008, aceptado para publicación el 21 de febrero de 2008.

Correspondencia a:

Raúl Escobar H.

E-mail: rescobar@med.puc.cl 
SHRN. Del total de SHRN, 83\% correspondió a causas centrales, destacando encefalopatía hipóxicoisquemica (ehi) (49\%) y genetopatía (15\%). Las causas periféricas correspondieron a 17\%, destacando hipermagnesemia (68\%) y miopatías (21\%). Conclusiones: Con los datos obtenidos, es posible concluir para este estudio: 1) El SHRN es una entidad clínica relativamente frecuente; 2) Las causas centrales son las más frecuentes; 3) Es posible estudio escalonado orientado a encontrar etiología específica del SHRN. (Palabras clave: síndrome hipotónico, hipotonía neonatal, recién nacido).

Rev Chil Pediatr 2008; 79 (2): 146-151

\section{Introducción}

El síndrome hipotónico del recién nacido (SHRN) se define como la disminución del tono muscular en las cuatro extremidades, tronco y cuello, durante los primeros 28 días de vida extrauterina ${ }^{1}$. El signo cardinal de este síndrome es la hipotonía, siendo este el principal hallazgo al examen físico. Es un cuadro aparentemente poco frecuente pero que plantea un problema diagnóstico importante.

La hipotonía en el recién nacido $(\mathrm{RN})$ puede estar presente como síntoma cardinal o principal, como ocurre en el SHRN, pero también puede ser uno del conjunto de signos y síntomas de un número importante de enfermedades propias de este grupo etáreo, siendo en este caso un signo clínico relativamente frecuente. La presencia de hipotonía per se, ya sea como signo principal o secundario, no tiene mayor relevancia en la evolución de la enfermedad causante de ella; su importancia radica en la potencial asociación a falta de fuerza.

El diagnóstico de hipotonía o tono anormalmente bajo durante el período neonatal es subjetivo y se basa en la experiencia del examinador. Al examinar un $\mathrm{RN}$, se debe tener en cuenta, entre otras cosas, la edad gestacional y el estado de sueño-vigilia durante el examen, esto último porque durante el sueño existe una disminución fisiológica del tono muscular, el que se acentúa más durante el sueño activo².

Si bien la presencia de hipotonía neonatal es un signo que se presenta en forma relativamente frecuente, hay escasa información en la literatura respecto a su prevalencia, la relación existente entre hipotonía y falta de fuerzas, y menos aún respecto a las causas etiológicas del SHRN. En los escasos reportes respecto a SHRN, se estima que el $80 \%$ corresponde a un origen central y dentro de éste la etiología más frecuente es la encefalopatía hipóxico isquémica, seguida por cuadros genéticos, malformaciones del SNC, alteraciones metabólicas, infecciones congénitas (TORCH) y endocrinopatías ${ }^{3,4}$. En cuanto al 20\% restante de SHRN, se considera que corresponden a afecciones del sistema nervioso periférico, estimándose que la mayoría de ellos corresponden a enfermedades musculares y con menor probabilidad a alteraciones de placa o de nervio periférico ${ }^{5}$.

Ante la presencia de un SHRN la decisión más importante a considerar es si se debe realizar una investigación diagnóstica en busca de la causa de la hipotonía o si se ha de posponer ésta esperando que la hipotonía sea transitoria, y si la decisión es estudiar, cual es la secuencia de exámenes más apropiada para esto.

De acuerdo a lo anterior, los objetivos de la presente comunicación fueron determinar la frecuencia de SHRN hospitalizados en la Unidad de Recién Nacidos del Hospital Clínico de la Pontificia Universidad Católica de Chile, determinar la causa etiológica en cada uno de los casos y proponer un método de aproximación diagnóstica.

\section{Pacientes y Métodos}

Se realizó un estudio retrospectivo a través de revisión de fichas clínicas, del período comprendido entre el $1^{\circ}$ de mayo de 2000 y el 30 de abril de 2006. Desde la base de datos de la Unidad de intermedio-intensivo de Neonatología del hospital clínico de la Pontificia Universidad Católica de Chile se ubicaron las fichas clínicas de los pacientes ingresados con diagnóstico, ya sea principal o secundario, de SHRN y de los pacientes que en sus diagnósticos de alta se consignó alguno de los diagnósticos o términos 
siguientes: hipomotilidad, falta de fuerzas, artrogriposis, arreflexia, falta de succión o esfuerzo respiratorio o atrofia muscular. Se revisó la ficha clínica de todos estos pacientes y se incluyó finalmente en el estudio a aquellos en que se consignó como signo clínico principal hipotonía, definida como la presencia de tono muscular por debajo de lo esperado para la edad gestacional, la que debió mantenerse por al menos 48 horas. Los pacientes portadores de síndrome de Down fueron registrados, pero aquellos diagnosticados precozmente y en los que no se planteó como diagnóstico SHRN ni hubo sospecha de otra entidad diagnostica como causante de su hipotonía, fueron excluidos del análisis final.

\section{Resultados}

Durante el período de estudio ingresó un total de 2158 recién nacidos, 168 de ellos con criterios de SHRN, lo que corresponde al 7,7\% del total. Cincuenta y cinco pacientes tuvieron el diagnóstico de síndrome de Down, todos ellos tuvieron un diagnóstico precoz, prenatal o postnatal inmediato, por lo que se excluyeron del análisis posterior.

En 113 casos, un 5,2\% del total, el motivo de ingreso principal o secundario correspondió a SHRN.

La edad gestacional promedio de los pacientes con SHRN fue de 36 semanas, con un rango de 25 a 41 semanas. Al agrupar a los pacientes por grupos de EG se puede observar que el mayor número de pacientes ingresados con el diagnóstico de SHRN corresponde al grupo de RN de 37 semanas y más, con un $66 \%$ del total (tabla 1).

Del total de SHRN, en el 83\%, que corresponde a 94 pacientes, la causa fue de origen central y en el 17\% (19 pacientes) la causa fue periférica.

Tabla 1. Clasificación de pacientes por EG

\begin{tabular}{lcc}
\hline EG & $\mathbf{n}$ & $\boldsymbol{\%}$ \\
\hline Menor o igual a 32 semanas & 12 & 10,6 \\
$33-36$ semanas & 26 & 23 \\
37 y más & 75 & 66,4 \\
Total & 113 & 100 \\
\hline
\end{tabular}

De las causas de origen central destacaron encefalopatía hipóxico isquémica (EHI) en 46 pacientes (49\%) y genetopatía en 14 pacientes (15\%), en éste último grupo destaca la trisomía 18 con 6 pacientes (tabla 2).

De las causas de origen periférico destacaron hipermagnesemia en 13 pacientes (68\%) y miopatía en 4 (21\%), estos correspondieron a distrofia muscular congénita en 2 casos, un paciente con miopatía nemalínica y un paciente con distrofia miotónica. Además, hubo un caso de miastenia congénita y 1 caso de enfermedad denervativa de extremidades inferiores, posiblemente una polineuropatia congénita (tabla 3).

En cuanto a la hipermagnesemia, los niveles de magnesio oscilaron desde 2,6 a 4,5 mg/dl y el

Tabla 2. Causas de SHRN de origen central

\begin{tabular}{lrc}
\hline Causa & n & \% \\
\hline EHI & 46 & 50 \\
Genetopatía & 14 & 15 \\
- Trisomía 18 & 6 & \\
- Prader Willi & 3 & \\
- Genetopatías no-especificadas & 2 & \\
- Síndrome dismórfico & 3 & \\
Malformación SNC & 11 & 12 \\
- Agenesia de cuerpo calloso & 1 & \\
- Hidranencefalia & 2 & \\
- Hidrocefalia & 2 & \\
- Mielomeningocele & 2 & \\
- Maformación SNC no especificada & 4 & \\
Factores maternos & & \\
(Drogas administradas FNB, DZP) & 3 & 3 \\
Metabólicas & 6 & 7 \\
Sepsis & 3 & 3 \\
Otras & 9 & 10 \\
\hline
\end{tabular}

Tabla 3. Causa de SHRN de origen periférico

\begin{tabular}{lcc}
\hline Causa & n & $\%$ \\
\hline Miopatía & 4 & 21 \\
DMC & 2 & \\
Miopatía nemalínica & 1 & \\
Distrofia miotónica congénita & 1 & \\
Hipermagnesemia & 13 & 69 \\
Miastenia congénita & 1 & 5 \\
Polineuropatía* & 1 & 5 \\
\hline * Diagnóstico probable, no confirmado & &
\end{tabular}

* Diagnóstico probable, no confirmado 
tiempo de hipotonía en relación a este factor en los pacientes afectados fue de máximo 72 horas, con recuperación completa posterior. No hubo una relación directa entre el nivel de magnesio en sangre y el grado de hipotonía.

Se logró llegar al diagnóstico definitivo del síndrome hipotónico en 106 casos de SHRN, 88 pacientes con SHRN central y 18 pacientes con SHRN periférico, en los restantes casos destacan 5 genetopatías no tipificadas, 1 paciente con sospecha clínica clara de enfermedad metabólica sin que se pudiese corroborar posteriormente y 1 paciente con enfermedad denervativa de extremidades inferiores, que presumiblemente correspondió a una polineuropatía congénita, pero que no continuó sus controles en nuestro hospital, por lo que su diagnóstico definitivo no pudo ser determinado.

\section{Discusión}

La hipotonía es un signo frecuente en el recién nacido, sin embargo, no es sinónimo de falta de fuerzas. Tal como describen distintos autores, los pacientes hipotónicos deben dividirse en aquellos que tienen falta de fuerza y aquellos que no, siendo la caracterización de los primeros los que tendrán implicancia en cuanto a diagnóstico y pronóstico ${ }^{6}$.

En nuestro estudio encontramos que la mayoría de los SHRN son de causa central, con proporciones similares a las descritas en la literatura, proporción que no ha variado en el tiempo a pesar de la mejoría tecnológica y los nuevos métodos diagnósticos disponibles.

De las causas centrales, la más frecuente en nuestra comunicación fue la asfixia neonatal, con $50 \%$ de los casos, al igual que los reportes extranjeros en los que este porcentaje llega a ser tan alto como del orden del $88 \%$, como se muestra en una de las series más grandes de síndrome hipotónico de Eng et $\mathrm{al}^{3}$, destacando eso sí que en este estudio se incluyeron pacientes con síndrome hipotónico de 0 a 3 años.

De las causas periféricas en nuestra casuística, la más frecuente después de la hipermagnesemia fueron las miopatías, contrario a lo reportado en la mayoría de los estudios extranjeros, en los que el primer grupo lo forman las enfermedades del asta anterior, con el síndrome de Werdning-Hoffman como causa principal, seguidos de patología muscular ${ }^{3,5}$. Esto puede deberse a la diferencia en el número y especialmente la edad de los pacientes analizados, ya que este trabajo abarca exclusivamente una cohorte de pacientes neonatos a diferencia de los estudios publicados en los que el rango de edad fue mayor. En el estudio de Richer et $\mathrm{al}^{3}$, en el cual se incluyen sólo RN, se encuentra una distribución porcentual similar a la encontrada por nosotros, lo que reafirma lo anteriormente mencionado.

En cuanto a la hipermagnesemia, esta fue una causa frecuente de hipotonía periférica, $\mathrm{y}$ que no siempre se menciona en la literatura. Niveles de magnesio superiores a 2,5 mg/dl se asociaron a SHRN, pudiendo el recién nacido tener niveles elevados de magnesio hasta después de los tres días de vida. Los niveles de magnesio elevados producen hipotonía por un defecto a nivel presináptico. Entre los principales factores de riesgo de hipermagnesemia neonatal están la administración a la madre de dosis altas de sulfato de magnesio por síndrome hipertensivo del embarazo, otros factores de riesgo son la prematurez y la asfixia neonatal severa. Los síntomas pueden ir desde rechazo en la alimentación, disminución de la motilidad intestinal con distensión abdominal, letargo, hipotonía, hiporeflexia, hasta apnea, por lo que es una causa de SHRN que se debe tener presente y manejarse precozmente.

Basándonos en nuestros hallazgos y en los escasos reportes de la literatura, es posible sugerir una aproximación escalonada al análisis de un recién nacido que se nos presente con un síndrome hipotónico. Una detallada historia prenatal, del parto y postnatal es esencial. Los antecedentes de convulsiones neonatales, clínica con evidencia de asfixia neonatal y la presencia de encefalopatía neonatal son altamente sugerentes de una causa central. Las neuroimágenes, incluyendo resonancia nuclear magnética (RNM) o tomogafía axial computada (TAC) de cerebro, son importantes herramientas para determinar si hay evidencia de alguna lesión cerebral que contribuya al cuadro de SHRN. Sin embargo, hay que tener presente que los trastornos periféricos también se pue- 
den presentar como asfixia neonatal hasta en un $28 \%$ de los casos, de estos la causa más frecuente son miopatías y menos frecuente los trastornos de nervio periférico ${ }^{7}$.

La presencia de hiporeflexia, hipomotilidad severa y contracturas, en ausencia de signos sugerentes de compromiso central, y asociado a gran compromiso respiratorio deben hacer pensar en una causa periférica.

La creatinfosfoquinasa (CK) plasmática elevada puede ser de gran utilidad para orientarse a una causa periférica de hipotonía, si bien en los primeros días de vida puede encontrarse elevada por otras causas, incluyendo el mismo parto. En nuestros pacientes fue de utilidad en la sospecha de una DMC, en los cuales la CK se mantuvo elevada más allá de las primeras 72 horas de vida del RN. La electromiografía (EMG) debe realizarse prontamente si se sospecha clínicamente un SHRN de causa periférica, puesto que permite una orientación diagnóstica rápida ${ }^{8}$, lo cual se demostró en este estudio en el cual la EMG permitió en 5 pacientes orientarse a un diagnóstico específico. En cuanto a la biopsia muscular y/o de nervio, esta es generalmente el último procedimiento diagnóstico en la evaluación de los pacientes con síndrome hipotónico, y permite el diagnóstico de condiciones musculares específicas, tales como distrofia muscular, miopatías metabólicas, por depósito o inflamatorias. Cuando la EMG entrega evidencias de un proceso miopático y/o la CK se mantiene persistentemente elevada, la indicación de biopsia muscular se debe considerar aún con mayor fuerza. En algunos pacientes y sólo si las condiciones clínicas lo permiten, se prefiere diferir la biopsa más allá del período de $\mathrm{RN}$, puesto que varias miopatías congénitas muestran variaciones a la histología que hacen su diagnóstico más fácil cuando el niño es mayor. Respecto a los estudios de biología molecular, estos no son indispensables en la mayoría de los casos de SHRN, sí deben ser considerados en aquellos pacientes en los cuales el diagnóstico no es posible de establecer con las técnicas diagnósticas ya descritas, o en etapas iniciales del estudio en aquellos pacientes en que la clínica plantea una alta sospecha diagnóstica. Un ejemplo de lo anterior a considerar es la distrofia miotónica en la que la mayoría de los pacientes con este trastorno tienen una madre afectada, por lo que el diagnóstico neonatal precoz puede sospecharse clínicamente ${ }^{9,10}$, como ocurrió en uno de los pacientes con SHRN periférico, quien tenía el antecedente de madre con distrofia miotónica y la biología molecular confirmó este diagnóstico.

Finalmente, debemos recalcar la importancia de intentar llegar a un diagnóstico definitivo en todos los casos de SHRN que se presenten con falta de fuerzas durante el período neonatal, ya que esto permite una mejor toma de decisiones respecto al soporte que deben recibir estos pacientes, permite entregar consejo genético y planificar adecuadamente la neurorehabilitación precoz para estos pacientes ${ }^{11}$.

\section{Conclusiones}

El SHRN es una entidad clínica relativamente poco frecuente en el período neonatal, siendo las causas de origen central las de mayor frecuencia. Los antecedentes y la observación clínica detallada son de vital importancia para la evaluación apropiada de estos pacientes y la toma de decisiones respecto a metodología de estudio. La causa más frecuente de SHRN en este grupo etáreo fue la encefalopatía hipóxico isquémica, seguido de genetopatías; mientras que entre las causas periféricas, el bloqueo de placa por sulfato de magnesio y las miopatías fueron las más frecuentes. La implementación de un esquema de evaluación progresivo en complejidad, siempre guiado por la historia y examen clínico, permiten llegar a un diagnóstico de certeza en la mayoría de estos pacientes.

\section{Referencias}

1.- Alfonso I, Papazian O, Valencia P: Hipotonía neonatal generalizada. Rev Neurol 2003; 37: 228-39.

2.- Kendall E, Schwartz J, Jessell T: Essentials of neural science and behavior. Appleton and Lange; 1995.

3.- Richer L, Shevell M, Miller S: Diagnostic profile of neonatal hypotonia: an 11-year study. Pediatr Neurol 2001; 25: 32-7.

4.- Johnston $H$ : The floppy weak infant revisited. Brain Dev 2003; 25: 155-8. 
5.- Dua T, Das M, Kabra M, et al: Spectrum of floppy children in indian scenario. Indian Pediatr 2001; 38: 1236-43.

6.- Dubowitz V: The floppy infant. Philadelphia: Lippincot Company 1980.

7.- Vasta I, Kinali M, Messinna S, et al: Can clinical signs identify newborns with neuromuscular disorders?. J Pediatr 2005; 146: 73-9.

8.- Jones HR: Electromyographic evaluation of the floppy infant. Pediatric clinical electromyography. Philadelphia: Lippincott-Raven Publishers 1996: 37-104.
9.- Erazo R: Hipotonía neonatal. Rev Neurol 2000; 31: 252-62.

10.- Verrijn S, Huisman M, Van Straaten H, Bakker J, Arabin B: "shake hands": diagnosing a floppy infantmyotonic dystrophy and the congenital Subtype: a difficult perinatal diagnosis. J Perinat Med 2000; 28: 497-501.

11.- Birdi K, Prasad A, Prasad C, Chodirker B, Chudley A: The floppy infant: retrospective analysis of clinical experience (1990-2000) in a tertiary care facility. J Child Neurol 2005; 20: 803-8. 